\title{
Once daily ceftriaxone and gentamicin for the treatment of febrile neutropenia
}

Richard J Tomlinson, Milind Ronghe, Colin Goodbourne, Christine Price, John S Lilleyman, Satya Das, Vaskar Saha

\begin{abstract}
Aims-To evaluate the pharmacokinetics of once daily (OD) gentamicin and its effectiveness as part of an OD regimen for the empirical treatment of febrile neutropenia in children with cancer.

Subjects-59 children aged 6 months to 16 years (mean (SD) 5.7 (4) years) with febrile neutropenia (neutrophil count $\left.<0.5 \times 10^{9} / 1\right)$ after chemotherapy.

Methods-Over one year, 113 febrile neutropenic episodes were treated empirically with an OD antibiotic regimen of ceftriaxone $(80 \mathrm{mg} / \mathrm{kg}$; maximum $4 \mathrm{~g})$ and gentamicin $(7 \mathrm{mg} / \mathrm{kg}$; infused over $60 \mathrm{~min}$ utes, no maximum). The patients were
\end{abstract} assessed after 48 hours.

Results-86 of the 113 episodes settled with the first line antibiotic regimen. In 29 episodes, blood cultures identified a causative bacterial pathogen; for 17 of these, the first line antibiotic regimen was adequate; in four episodes, although the episode settled, ceftriaxone was replaced by a more appropriate antibiotic and OD gentamicin was continued; in the remaining eight episodes, a glycopeptide antibiotic was deemed necessary. There was no failure of treatment in organisms sensitive to gentamicin, including Pseudomonas aeruginosa. In 27 episodes $(24 \%)$, resolution was obtained by the empirical introduction of a second line regimen of ceftazidime and a glycopeptide antibiotic, and/or amphotericin. Gentamicin concentrations were measured in 110 episodes and they were all below the 24 hour line indicating that there was no need to change the dosing interval. In two episodes $(2 \%)$, serum creatinine rose transiently by more than $50 \%$ of the baseline concentration. Although there was no vestibular toxicity, three of 30 children who underwent pure tone audiometry reported high frequency hearing loss in one ear.

Conclusion-OD gentamicin can be used safely and effectively to treat febrile neutropenia in children with cancer. When used for a short period ( $<5$ days), in children not receiving other nephrotoxic drugs and who have normal serum creatinine, serum gentamicin estimations are unnecessary.

(Arch Dis Child 1999;80:125-131)

Keywords: once daily treatment; febrile neutropenia; ceftriaxone; gentamicin; cancer
Severe neutropenia is a common complication of the intensive chemotherapeutic regimens used to treat most childhood cancers. After chemotherapy, neutropenia can persist from a few days to a few weeks. During this time, the child is vulnerable to infections and may develop a fever with few or no localising signs. Such children with febrile neutropenia often fail to mount an adequate inflammatory response to infectious agents, ${ }^{1}$ and are at a high risk of developing life threatening infections. ${ }^{1}$ In up to $40 \%$ of these cases a causative bacterial pathogen can be isolated from cultures. ${ }^{2}$ These organisms tend to be those that colonise normal or damaged skin and mucosal surfaces and are either Gram negative (such as Escherichia coli, pseudomonas, klebsiella, and enterobacter) or Gram positive (such as Staphylococcus aureus, coagulase negative staphylococcus, and enterococcus). ${ }^{3}$ Because culture results are not obtainable at presentation, initial treatment is almost always empirical. ${ }^{1}$ In the past, monotherapy with either ceftriaxone ${ }^{4}$ or ceftazidime ${ }^{5}$ has been used successfully to treat children with febrile neutropenia. However, animal studies suggest that in patients with neutropenia and Gram negative septicaemia, a combination of $\beta$ lactam and aminoglycoside antibiotics offers increased bactericidal activity, synergism, and a broader antibacterial spectrum. This also limits the emergence of resistance with fewer superinfections and increases the post-antibiotic effects of both drugs. ${ }^{6-9}$ Based on this understanding, in common with many other units, our unit policy has been to use thrice daily ceftazidime and gentamicin as an empirical treatment for febrile neutropenia. This combination has the advantage of having a broad spectrum activity against Pseudomonas aeruginosa. Although this has proved to be satisfactory, its disadvantage is that it has to be given three times a day and trough and peak serum concentrations of gentamicin have to be estimated. In an analysis of workload, up to $40 \%$ of our staff time was being consumed in administering antibiotics. Therefore, although this regimen was effective, it was burdensome. What was required was less frequent administration of antibiotics, ideally on a once a day basis and a regimen that required little or no monitoring.

For the reasons mentioned above, we elected to continue with a combination of $\beta$ lactam and aminoglycoside antibiotics as empirical treatment. During the preceding three years, 212 $(23 \%)$ of 925 bacterial isolates obtained from children in our unit were of Gram negative bacteria and only $12(1 \%)$ were identified as 
Table 1 A comparison of published reports using once daily aminoglycosides for the empirical treatment of febrile neutropenia in children with cancer

\begin{tabular}{llllllll}
\hline Frequency & Antibiotic regimen & $\begin{array}{l}\text { No of } \\
\text { episodes }\end{array}$ & $\begin{array}{l}\text { Blood culture } \\
\text { positivity (\%) }\end{array}$ & $\begin{array}{l}\text { Response } \\
\text { rate (\%) }\end{array}$ & $\begin{array}{l}\text { Ototoxicity } \\
\text { (\%) }\end{array}$ & $\begin{array}{l}\text { Nephrotoxicity } \\
\text { (\%) }\end{array}$ & Reference \\
\hline Once daily & CF + Amik & 350 & 23 & 71 & 9 & 3 & $19 \star$ \\
Twice daily & CZ + Amik & 344 & 26 & 74 & 7 & 2 & 19 \\
Once daily & CF + Amik & 181 & 26 & 66 & 2 & $<1$ & 21 \\
Twice daily & CZ + Amik & 183 & 29 & 66 & 1 & $<1$ & 21 \\
Once daily & Carb + Amik & 25 & NA & 96 & 0 & 0 & 24 \\
Twice daily & Carb + Amik & 25 & NA & 92 & 0 & 0 & 23 \\
Once daily & Pip + Genta & 30 & 16 & 90 & NA & 0 & 23 \\
Twice daily & Pip + Genta & 22 & 18 & 91 & NA & 0 & NA \\
Once daily & CF + Amik + Teico & 296 & 26 & 92 & $? 0$ & NA & 22 \\
Once daily & CF + Amik & 990 & NA & 93 & NA & 2 & Present study \\
Once daily & CF + Genta & 113 & 26 & 74 & $? 10$ & & 23 \\
\hline
\end{tabular}

*This study included both adults and children.

CF, ceftriaxone; Amik, amikacin; Carb, carbenicillin (TD); Pip, piperacillin (QD); Genta, gentamicin; Teico, teicoplanin; NA, not available.

pseudomonas. Because the vast majority of isolates were either Gram positive, or nonpseudomonal Gram negative organisms, we reasoned that ceftriaxone would be an acceptable alternative to ceftazidime. Although it has little antipseudomonal activity, it has excellent activity against other Gram negative rods and, when compared with ceftazidime, better activity against $S$ aureus and Staphylococcus epidermidis. ${ }^{9}{ }^{10}$ Because ceftriaxone is given as a once daily (OD) dose, it would be ideal also to administer the aminoglycoside as an OD dose, to maximise the advantage of once daily treatment.

Considerable evidence exists for using aminoglycosides on an OD basis. The efficacy of aminoglycosides is related to the area under the plasma concentration time curve and is thus independent of the dosing frequency. ${ }^{11} \mathrm{~A}$ peak concentration:minimum inhibitory concentration (MIC) ratio of at least $8: 1$ to $10: 1$ is necessary for optimal bactericidal activity and a higher peak concentration can lead to better outcomes. ${ }^{12}$ Bacteria that survive the initial dose develop a time dependent failure of the transport mechanism required to transport the drug into the bacterial cell. ${ }^{13}$ Because the aminoglycoside is unable to gain entry to the inside of the cell during this time, the organism becomes temporarily resistant to the drug. This is known as adaptive resistance. In the continued presence of the drug, adaptive resistance is enhanced and prolonged, in its absence, the organism is soon able to return to its normal phenotype. Maximal adaptive resistance appears to occur between eight and 16 hours after exposure, suggesting that the dosing interval needs to be at least 24 hours. ${ }^{14}$ Therefore, frequent dosage is unlikely to increase bacterial kill and more likely to promote adaptive resistance. Although higher peak concentrations increase the post-antibiotic effect they might also decrease toxicity. Renal toxicity and ototoxicity of aminoglycosides appear to be related to the duration of exposure rather than the peak concentration of drug. ${ }^{15-17}$ Therefore, it would seem logical to use an OD dosing of aminoglycoside, rather than the conventional thrice or twice daily (TD) regimen. The clinical efficacy and toxicity of OD aminoglycosides have been reported in a variety of adult populations including cancer patients with neutropenia. Meta-analysis performed on these studies suggests that OD aminoglycosides are at least as effective and certainly no more toxic than the conventional TD dosage. ${ }^{18}$ At least six published studies have evaluated the efficacy of OD aminoglycosides in children with febrile neutropenia ${ }^{19-24}$ (table 1). They all found OD dosing to be as safe and as clinically effective as TD administration. While one study evaluated the pharmacokinetics of OD gentamicin $^{23}$ the others have evaluated the efficacy of OD amikacin. ${ }^{19-22} 24$ Thus, we felt that there was sufficient evidence for the use of OD aminoglycosides in the treatment of children with febrile neutropenia. However gentamicin, not amikacin, is the cheapest and most widely used of the aminoglycosides and there is insufficient information available regarding the optimal regimen and criteria for its use on an OD basis in children. In our paper we report on the safety and efficacy of an OD antibiotic regimen using OD gentamicin and ceftriaxone for the empirical treatment of febrile neutropenia.

\section{Patients and methods}

From July 1996, all children admitted to the paediatric oncology unit at St Bartholomew's Hospital with a diagnosis of febrile neutropenia after chemotherapy were considered eligible for inclusion in the study. For purposes of the study, neutropenia was defined as an absolute neutrophil count of $<0.5 \times 10^{9} / 1$. Fever was defined as a single axillary recording of $>38.5^{\circ} \mathrm{C}$ or a persistently raised temperature of $>38^{\circ} \mathrm{C}$ over a four hour period. At admission, all children were assessed clinically and baseline investigations including blood culture, serum creatinine, and a full blood count were performed. Those who presented in shock, with a raised serum creatinine or a documented pseudomonal infection within the preceding three months, were excluded. Those with ongoing oral prophylaxis with antibacterial, antifungal, or antiviral agents were not excluded. The study group was treated empirically with an OD regimen of ceftriaxone and gentamicin. Ceftriaxone was given at a dose of $80 \mathrm{mg} / \mathrm{kg}$, with a maximum dose of $4 \mathrm{~g}$ as a slow intravenous bolus. Gentamicin was administered at a dose of $7 \mathrm{mg} / \mathrm{kg}$, with no maximum dose, infused intravenously in $50-100 \mathrm{ml}$ of $0.9 \%$ saline over 60 minutes. Gentamicin concentrations were analysed by immunoassay 
six to 14 hours after the start of the first infusion and at five day intervals if still receiving gentamicin. Values were interpreted on the Hartford nomogram ${ }^{25}$ and the dose interval was adjusted if necessary. The patients were reassessed at the end of 48 hours and fell into four groups. Group A, where fever had subsided and cultures were negative received antibiotics for a total of 48 afebrile hours and patients were then allowed home if clinically well. Group B, where fever had subsided but cultures were positive for an organism sensitive to either or both of the antibiotics received one or both antibiotics for a minimum of seven days in Gram positive and 10 days in Gram negative infections. Group C comprised children in whom fever did not resolve after 48 hours and cultures identified an organism resistant to the first line antibiotics; for these children, antibiotic(s) appropriate to the sensitivity of the organism were started. Group D comprised children in whom there was no resolution of fever at the end of 48 hours and cultures remained sterile. In these patients, ceftriaxone and gentamicin were stopped and they were started on thrice daily ceftazidime, and twice daily vancomycin, or daily teicoplanin. Intravenous amphotericin was given if there was evidence of invasive fungal infection, or if the fever persisted beyond five days. Additional antibiotics were used for localised infections and aciclovir was given for severe mucositis.

To assess for gentamicin associated toxicity, serum creatinine was monitored during the period of hospitalisation, and at least once at a later date. Nephrotoxicity was defined as a rise in the serum creatinine of more than $50 \%$ of the baseline value. ${ }^{12}$ Vestibular toxicity was defined as the development of vertigo with nausea and/or vomiting, gait disturbances, or nystagmus. Normal otoscopic examination was carried out to exclude external and middle ear problems and pure tone audiometry was then performed on all children of a cooperative age after a minimum of two weeks off treatment. Ototoxicity was defined as a decline in the auditory or vestibular inner ear function with no discernible cause. Audiograms were considered to be abnormal if acuity at any test frequency $(0.25,0.5,1,2,4$, or $8 \mathrm{kHz})$ in one or both ears decreased by $>20 \mathrm{~dB}$. To determine the applicability of the Hartford nomogram in our study group, serum gentamicin measurements were performed at 0.5 , 1,3 , and 8 hours after the start of the infusion of the first dose of gentamicin. This was

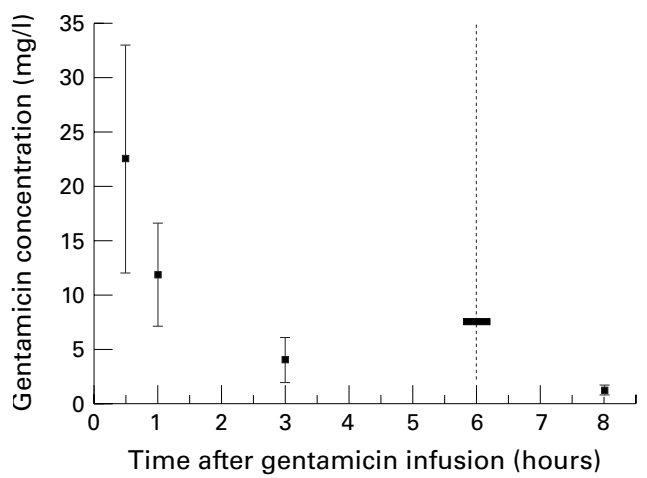

Figure 1 Mean serum concentrations of gentamicin at $0.5,1,3$, and 8 hours after $7 \mathrm{mg} / \mathrm{kg}$ was infused over 60 minutes in 20 children. Filled squares represent mean values and lines represent 2 SDs from the mean. The dotted line at 6 hours shows the start of the Hartford nomogram, the bar indicates the gentamicin concentration above which the dosing interval needs to be increased.

performed in 20 children, with a normal serum creatinine, picked to represent yearly age intervals from the ages of $0.5-12$ years, and in whom we were able to obtain a verbal consent to carry out the pharmacokinetic profile.

\section{Results}

From July 1996 to July 1997, 75 children aged between 6 months and 16 years (median, 5 years) with 144 febrile episodes were considered for the OD first line treatment. A total of 31 episodes were excluded from the study; 13 fulfilled the exclusion criteria and 18 had a neutrophil count higher than 0.5 . Therefore, a total of 113 episodes of febrile neutropenia in 59 children ( 31 boys and 28 girls) aged between 6 months and 16 years (mean (SD) 5.7 (4) years) were analysed. The study group consisted of 33 children with acute lymphoblastic leukaemia (ALL); five children with acute myeloid leukaemia (AML); six children with retinoblastoma; five children with nonHodgkin's lymphoma; three children with neuroblastoma; two children each with nephroblastoma and rhabdomyosarcoma; and three children with other tumours. Of this group, four children (two each with ALL and AML) were in their nadir, post-allogeneic bone marrow transplantation.

A gentamicin pharmacokinetic profile was assessed in 20 children with ages ranging from 1 to 12 years. Initially, we aimed to have an equal number of children in each year age group; however, this was not possible. We were unable to recruit anyone between the ages of 5-6 years of age. There were four children in

Table 2 Clinical response after 48 hours of empirical treatment with once daily ceftriaxone and gentamicin in 113 febrile neutropenic episodes

\begin{tabular}{lllll}
\hline & Number & $\begin{array}{l}\text { Days on } \\
\text { gentamicin } \\
\text { (median) }\end{array}$ & $\begin{array}{l}\text { Days on } \\
\text { antibiotics } \\
\text { (median) }\end{array}$ & Antibiotics received \\
\hline $\begin{array}{l}\text { Single daily ceftriaxone and gentamicin } \\
\text { Group }\end{array}$ & & & & \\
A Afebrile and culture negative & 60 & 4 & 4 & ODG, CF \\
B $\quad$ Afebrile and culture positive & 17 & 7 & 7 & ODG, CF, others \\
C Febrile and culture positive & 7 & 2 & 6 & CF and ODG then CFZ, VAN, \pm Am \\
D $\quad$ Febrile and culture negative & 20 & 2 & 6 & CF and ODG then CFZ, VAN, \pm Am \\
Single daily gentamicin with another antibiotic \\
$\quad$ Afebrile culture negative & 4 & 4 & 4 & ODG, CFZ \\
$\quad$ Afebrile culture positive & 5 & 5 & 5 & ODG, others \\
\hline
\end{tabular}

ODG, once daily gentamicin; CF, ceftriaxone; CFZ, ceftazidime; VAN, vancomycin or teicoplanin; Am; amphotericin or ambisome; others, CFZ (2), VAN (1), flucloxacillin (2). 
Table 3 Bacterial culture isolates in 29 of 113 febrile neutropenic episodes and the antibiotic regimen used to treat the infection

\begin{tabular}{llc}
\hline Antibiotic regimen & Organism & Number \\
\hline Single daily gentamicin and ceftriaxone & Escherichia coli & 4 \\
& Klebsiella & 3 \\
& Klebsiella and enterobacter & 1 \\
& Streptococcus & 3 \\
& & \\
Ceftriaxone changed electively & Klebsiella and enterobacter & 1 \\
& Pseudomonas aeruginosa & 1 \\
& Salmonella & 1 \\
& B Haemolytic streptococci & 1 \\
Antibiotics changed due to culture results & & 4 \\
& Coagulase negative staphylococcus & 1 \\
& $\alpha$ Haemolytic streptococci & 1 \\
& Streptococcus acidominimus & 1 \\
& Stenotrophomonas & 2 \\
Single daily gentamicin with other cephalosporins & Mixed organisms & 2 \\
& Staphylococcus aureus & 1 \\
& Enterococcus faecalis & 1 \\
& Klebsiella & 1 \\
Total & Coliform and streptococcus & 29
\end{tabular}

the $<2$ year age group, three each in the $2-3$ and 4-5 year age groups, two each in the 11-12 and over 12 years of age groups, and one each in the other age groups. As seen in fig 1 , after a single intravenous infusion of $7 \mathrm{mg} / \mathrm{kg}$ over 60 minutes, peak serum concentrations of 5.2$37.2 \mathrm{mg} / \mathrm{kg}$ were seen between 30 minutes and one hour. All values fell below the 24 hour line of the Hartford nomogram by eight hours.

Table 2 shows the response to treatment. A total of $86(76 \%)$ of the 113 episodes settled with the first line antibiotic treatment. These include those in groups $\mathrm{A}$ and $\mathrm{B}$ and the nine episodes treated with OD gentamicin but a cephalosporin other than ceftriaxone. If these nine are excluded, 77 (74\%) of the 104 episodes responded to a single daily regimen of ceftriaxone and gentamicin. In the remaining $27(24 \%)$ in groups C and D, the episode settled after second line treatment with ceftazidime and vancomycin, with or without amphotericin. In the nine episodes where a cephalosporin other than ceftriaxone was used, four responded to the empirical combination of thrice daily ceftazidime and OD gentamicin, and no pathogen was isolated. As shown in table 3, in 29 (25\%) episodes, one or more organisms were isolated from blood cultures.

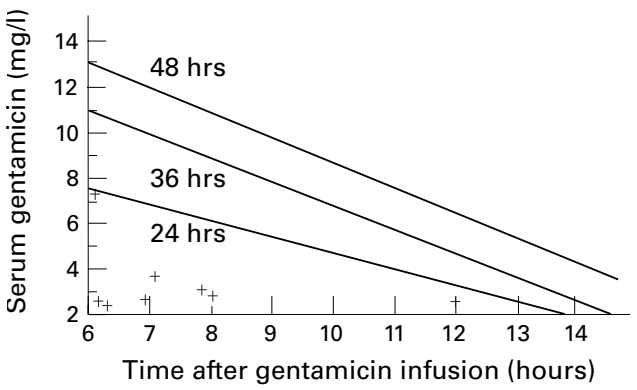

Figure 2 The Hartford nomogram for calculating the interval between dosages with OD gentamicin. Serum concentrations are estimated 6 to 14 hours after the start of the gentamicin infusion and plotted on the graph. The position of the value in relation to the baseline and the 24, 36 , and 48 hour lines indicates the dosage frequency. Of 110 values obtained, 102 were $<2.0 \mathrm{mg} / \mathrm{ml}$ and cannot be shown on the graph. The remaining eight values obtained are shown as crosses. All values obtained were consistent with a 24 hour dosage interval.
For the five culture positive episodes (tables 2 and 3) where a cephalosporin other than ceftriaxone was used, two had clinical evidence of catheter tunnel infection and blood cultures grew $S$ aureus. Flucloxacillin along with OD gentamicin had been prescribed at the start of each episode. In one episode, a perineal abscess was detected, vancomycin, OD gentamicin, and metronidazole were commenced and Enterococcus faecalis sensitive to vancomycin was isolated. In the other two episodes, children received ceftazidime and gentamicin because of the physicians' bias, but the isolated organisms were sensitive to ceftriaxone as well as ceftazidime and gentamicin. Of the remaining 24 blood culture positive episodes, 17 were afebrile within 48 hours of first line treatment (group B; table 2). Of these, 11 (table 3) were sensitive to ceftriaxone and/or gentamicin and treated successfully with first line antibiotics. As shown in table 3, in an additional four episodes, although the isolated organism showed in vitro sensitivity and the child responded clinically, ceftriaxone was stopped electively and replaced with another antibiotic while OD gentamicin was continued. In the klebsiella and pseudomonas isolates, ceftazidime was substituted as it was felt that this would be more appropriate cover. The episode with salmonella septicaemia was treated with additional ciprofloxacin and the $\beta$ haemolytic streptococcus with penicillin. In the nine episodes (table 3 ) where the antibiotics were changed as a result of the sensitivity of the isolate, this was because of ceftriaxone resistant isolates in eight. Although the Streptococcus acidominimus isolate was sensitive to ceftriaxone (and indeed responded clinically), it was felt that a glycopeptide antibiotic would be more effective. One mixed isolate consisting of $E$ coli and coagulase negative staphylococcus also showed clinical improvement, but the ceftriaxone was replaced with teicoplanin and the OD gentamicin was continued.

The absolute dose of gentamicin prescribed ranged from 50 to $350 \mathrm{mg}$. Table 2 shows the median length of gentamicin treatment in the various groups. As shown in fig 2, gentamicin values were obtained in 110 episodes and when plotted on the Hartford nomogram they were all below the 24 hour line, indicating that there was no need for a change in the dose interval. All but one value ranged between $<1$ and $3.8 \mathrm{mg} / \mathrm{l}$. A concentration of $7.2 \mathrm{mg} / 1$ in one episode taken at six hours was considered borderline/normal but we electively chose to alter the dosage interval to 36 hourly. A subsequent value taken eight hours after infusion was found to be $3.1 \mathrm{mg} / 1$. In three episodes, a gentamicin value was not obtained because venous access failed, and in all these cases the children improved within 48 hours and the gentamicin was stopped within 96 hours. In 17 episodes, gentamicin was continued beyond five days, and in all cases the five day serum gentamicin values were less than $3.8 \mathrm{mg} / \mathrm{l}$. The mean serum creatinine for the study population was unchanged before and after gentamicin treatment, at $52 \mu \mathrm{mol} / \mathrm{l}$. In two episodes, a transient rise in serum creatinine of 
$50 \%$ was recorded. These children were treated electively with gentamicin at 36 hourly intervals without complications, required treatment for less than five days, and in both instances the serum creatinine returned to normal within 14 days. During gentamicin treatment no child reported tinnitus or hearing loss and none were found to have nystagmus or gait problems. Pure tone audiometry was performed on all cooperative children over the age of 4 years at least two weeks after receiving OD gentamicin treatment. Of 30 children assessed, 27 audiograms were normal and three fulfilled possible ototoxicity criteria by having a $20-30 \mathrm{~dB}$ loss in one ear at one frequency.

\section{Discussion}

This was a prospective, observational study rather than a randomised trial because the efficacy of OD aminoglycoside in febrile neutropenia has already been established. Our objectives were to study the pharmacokinetics of OD gentamicin and establish a dosage and monitoring schedule. We were also concerned about the efficacy of the regimen and table 1 shows the comparison between our results and those published recently from other trials in children with febrile neutropenia. The first four studies compared the use of an OD regimen with a twice daily amikacin regimen, and the other two studies reported on the efficacy of an OD regimen. Only one group reported on the use of OD gentamicin, although this was reported as a pharmacokinetic profile rather than a clinical trial. Because there was no reason to suppose that the experience with gentamicin would be any different from that seen with amikacin, we chose not to carry out a comparative trial. The hospital antibiotic monitoring group agreed with us that there was sufficient data to prescribe gentamicin safely as a single daily dose to children with febrile neutropenia. Parents were informed about the change in the antibiotic policy and involved in the monitoring for toxicity. As seen in table 1 , the efficacy and toxicity results of the OD gentamicin regimen in our population are comparable with those seen in other studies using OD aminoglycosides. Our results are also comparable to those reported for TD aminoglycosides, although this was not evaluated directly in our study.

The recommendations for dosage in OD gentamicin regimens in children have ranged from 5 to $10 \mathrm{mg} / \mathrm{kg} /$ day. ${ }^{23}$ Patients with cancer are thought to have an increased volume of drug distribution leading to reduced drug concentration. ${ }^{27}{ }^{28}$ Higher than standard dosages of gentamicin might be required if therapeutic serum concentrations are to be obtained. ${ }^{26}$ The Hartford study, ${ }^{25}$ standardised in an adult population, recommended a gentamicin dose of $7 \mathrm{mg} / \mathrm{kg}$ infused over 60 minutes. Because we chose to use the nomogram devised in that study to evaluate the dose interval, we also used the same dose and infusion time. A comparison between figs 1 and 2 shows that the parameters recommended by the Hartford nomogram were applicable for use in our population. The recommended monitoring using OD gentamicin is to measure the trough serum concentration. It is this concentration that identifies potential toxicity because a higher than expected value reflects the kidney's inability to excrete the drug. In practice, we found no child with a normal creatinine who required a dosage adjustment. In children receiving OD gentamicin and not receiving any other nephrotoxic drug and with normal serum creatinine concentrations, we now believe that the measurement of trough serum concentrations is unnecessary. Because gentamicin is the cheapest of the available aminoglycosides we feel that this will be a practical issue where resources are limited. The OD regimen allowed us to introduce outpatient treatment in selected patients and relieved pressure on inpatient beds and met with the approval of patients and families. Prescribing was made easier because the dose does not need to be changed and only the dosage interval is adjusted.

Unfortunately, this study does not provide data for the treatment of patients with impaired renal function. When the serum creatinine is deranged, one possibility is to reduce the dose to permit the use of a 24 hour dosing schedule. ${ }^{12}$ However, the nomogram recommends that in a patient with a raised serum creatinine, the full dose should be given and the dose interval adjusted according to the gentamicin concentration. In those patients with severe renal failure (creatinine clearance $<20 \mathrm{ml} /$ minute) serial measurements should be performed to determine the time of the next dose. The latter approach is more practical because in our patient population the serum creatinine is usually available only after the first dose of gentamicin. If a child is anuric or known to have impaired renal function, we avoid the use of empirical aminoglycosides. If the sensitivity of bacterial isolates is such that an aminoglycoside is the only drug of choice in such children, our unit policy is to use the full dose and adjust the dose interval using the nomogram. We have not had an opportunity to test this in practice and because of a lack of data have no way of proving whether this is the best approach.

One of the arguments raised against the use of OD aminoglycosides in children with neutropenia is that for most of the day serum and tissue aminoglycoside concentrations are below the minimal inhibitory concentration of the infecting organism. During this time interval, the compromised immune system cannot be relied upon to help eradicate the pathogen. The dose of OD gentamicin might need to be as high as $10 \mathrm{mg} / \mathrm{kg}$ to have the desired effect ${ }^{26}$ and even then the post-antibiotic effects might be insufficient to eradicate the infecting organism. ${ }^{29}{ }^{30}$ In our study, of the 34 organisms isolated, 17 were Gram negative. There was no failure of treatment in organisms sensitive to gentamicin (recurrence of infection with the same organism once treatment was stopped). There was only one case of pseudomonas septicaemia and this was treated initially with the single daily regimen. Although the child responded clinically within 24 hours, on know- 
ing the culture results we changed the ceftriaxone to TD ceftazidime but continued giving the gentamicin as an OD dose. Therefore, clinically, an adequate post-antibiotic effect of gentamicin was seen in Gram negative infections occurring in our study population, although it is likely that this was enhanced by the concomitant use of a cephalosporin antibiotic. ${ }^{31}$ This observation is supported by other studies (table 1), where the response rate has ranged from $66-96 \%$.

Renal toxicity was minimal. Included in the analysis were two children with subtotal nephrectomies and they did not have a rise in their serum creatinine. In the two children who showed a rise in their serum creatinine while on OD gentamicin, values quickly returned to normal. No vestibular toxicity was detected. It was not practically possible for us to perform audiograms on children before they received OD gentamicin and the three children with borderline high frequency hearing defects had all received other ototoxic agents. Therefore, we cannot be sure that this hearing defect was caused by OD gentamicin. However, it is reassuring that even if this is the case the numbers are very small. In general, most studies have either not shown any difference in toxicity between OD and TD aminoglycosides or decreased toxicity with OD administration. ${ }^{12} 1921-2532$

At the beginning of our study, children who presented with shock or those who had a recent history of pseudomonas infection were excluded. However, the first four children who presented in shock were subsequently shown to have Gram positive infections. At present, gentamicin is being prescribed only as an OD dose in our unit, irrespective of the child's condition. At the time of going to press, we have given OD gentamicin in over 300 episodes, safely and effectively. Although some studies have recommended additional Gram positive cover with glycopeptide antibiotics, ${ }^{20}{ }^{33}$ we feel that this is unnecessary and possibly dangerous. About $20-30 \%$ of children with febrile neutropenia will require glycopeptide antibiotics, mostly because of problems with indwelling central venous lines related to coagulase negative staphylococci. ${ }^{34}$ As is illustrated by our study, although associated with morbidity, these infections are rarely associated with mortality, and it is safe to wait for a therapeutic response or results of cultures, unless clinically indicated. The worry is the increasing emergence of glycopeptide resistant, Gram positive organisms as a result of inappropriate usage of these antibiotics, ${ }^{35}$ and perhaps these antibiotics should not be used for first line, empirical treatment. $^{36} 37$ Similarly, trials evaluating shorter aminoglycoside dosing intervals ${ }^{38}$ have little therapeutic advantage and might facilitate the development of resistance.

Our study shows that OD gentamicin, given as an infusion, can be used safely and effectively for the empirical treatment and management of severe sepsis in children with neutropenia and cancer. There are still some unanswered questions of practical importance. A more complete pharmacokinetic study needs to be carried out to validate the Hartford nomogram in children. We also need to evaluate the dose and infusion schedules to see if they are optimal for the paediatric population - that is, whether peak gentamicin concentrations of 8-10:1 of the MIC are being achieved. If OD gentamicin can be given as a bolus dose, rather than as an infusion, it will then be possible to treat many more children on an outpatient basis. Additional pharmacokinetic studies are required to examine this possibility, although the advent of liposomal formulations ${ }^{39}$ might make this the more practical approach.

We thank Dr J E Kingston for her support. Some of these data were presented at the 29th International Society of Paediatric Oncology meeting, Istanbul, 23-27 September 1997.

1 Pizzo PA, Rubin M, Freifeld A, Walsh TJ. The child with cancer and infection. I. Empiric therapy for fever and neu-
tropenia, and preventive strategies. F Pediatr 1991;119:679tropen.

2 Klastersky J, Zinner SH, Calandra T, et al. Empiric antimicrobial therapy for febrile granulocytopenic cancer patients: lessons from four EORTC trials. Eur $\mathcal{F}$ Cancer Clin Oncol 1988;24(Suppl 1):S35-45.

3 Klastersky J. Therapy for sepsis in neutropenic patients. In: Sande MA, Root RK, eds. Treatment of serious infections in the 1990s. Contemporary issues in infectious diseases. Edinburgh: Churchill Livingstone, 1992:47-63.

4 Smith L, Will AM, Williams RF, Stevens RF. Ceftriaxone vs. azlocillin and netilmicin in the treatment of febrile neutropenic children. F Infect 1990;20:201-6.

5 Pizzo PA, Hathorn JW, Hiemenz J, et al. A randomized trial comparing ceftazidime alone with combination antibiotic therapy in cancer patients with fever and neutropenia. $N$ therapy in cancer patients with
Engl f Med 1986;315:552-8.

6 Buxbaum A, Georgopoulos A. Postantibiotic effect of ceftriaxone and gentamicin alone and in combination on Klebsiella pneumoniae, Pseudomonas aeruginosa and Streptosiella pneumoniae, Pseudomonas aerugin

7 Kapusnik JE, Hackbarth CJ, Chambers HF, Carpenter T, Sande MA. Single, large, daily dosing versus intermittent dosing of tobramycin for treating experimental Pseudomonas pneumonia. F Infect Dis 1988;158:7-12.

8 Hathorn JW, Pizzo PA. Is there a role for monotherapy with beta-lactam antibiotics in the initial empirical management of febrile neutropenic cancer patients? F Antimicrob Chemother 1986;17(Suppl A):41-54.

9 Goldstein FW, Pean Y, Gertner J. Resistance to ceftriaxone and other beta-lactams in bacteria isolated in the community. The Vigil'Roc study group. Antimicrob Agents Chemother 1995;39:2516-19.

10 Fuchs PC, Barry AL, Brown SD. Survey of antimicrobial activity of four commonly used third generation cephalosporins tested against recent bacterial isolates from ten American medical centers, and assessment of disk diffusion test performance. AST surveillance group. Diagn Microbiol Infect Dis 1996;24:213-19.

11 Roosendaal R, Bakker-Woudenberg IA, van den Berghe-van Raffe M, Michel MF. Continuous versus intermittent administration of ceftazidime in experimental Klebsiella pneumoniae pneumonia in normal and leukopenic rats. Antimicrob Agents Chemother 1986;30:403-8.

2 Freeman CD, Nicolau DP, Belliveau PP, Nightingale CH. Once-daily dosing of aminoglycosides: review and recommendations for clinical practice. $\mathcal{F}$ Antimicrob Chemother 1997;39:677-86.

13 Daikos GL, Lolans VT, Jackson GG. First-exposure adaptive resistance to aminoglycoside antibiotics in vivo with meaning for optimal clinical use. Antimicrob Agents Chemother 1991;35:117-23.

14 Xiong YQ, Caillon J, Kergueris MF, et al. Adaptive resistance of Pseudomonas aeruginosa induced by aminoglycosides and killing kinetics in a rabbit endocarditis model. Antimicrob Agents Chemother 1997;41:823-6.

15 Verpooten GA, Giuliano RA, Verbist L, Eestermans G, De Broe ME. Once-daily dosing decreases renal accumulation of gentamicin and netilmicin. Clin Pharmacol Ther 1989;45: $22-7$

16 Swan SK. Aminoglycoside nephrotoxicity. Semin Nephrol 1997;17:27-33.

17 Kuharcik CM, Craig AC. New trends in aminoglycoside dosing. Anna fournal 1994;21:447-8.

18 Gilbert DN. Meta-analyses are no longer required for determining the efficacy of single daily dosing of aminoglycosides. Clin Infect Dis 1997;24:816-19.

19 Anonymous. Efficacy and toxicity of single daily doses of amikacin and ceftriaxone versus multiple daily doses of amikacin and ceftazidime for infection in patients with cancer and granulocytopenia. The international antimicrobial therapy cooperative group of the European organization for research and treatment of cancer. Ann Intern Med tion for research a 
20 Bouffet E, Fuhrmann C, Frappaz D, et al. Once daily antibiotic regimen in paediatric oncology. Arch Dis Child 1994, biotic regimer.

21 Charnas R, Luthi AR, Ruch W. Once daily ceftriaxone plus amikacin vs. three times daily ceftazidime plus amikacin for treatment of febrile neutropenic children with cancer. Writing committee for the international collaboration on antimicrobial treatment of febrile neutropenia in children. Pediatr Infect Dis $\mathcal{F}$ 1997;16:346-53.

22 Sahu S, Bapna A, Pai SK, Nair CN, Kurkure PA, Advan $\mathrm{SH}$. Outpatient antimicrobial protocol for febrile neutropenia. Pediatr Hematol Oncol 1997;14:205-11.

23 Postovsky S, Arush MWB, Kassis E, Elhasid R, Krivoy N. Pharmacokinetic analysis of gentamicin thrice and single daily dosage in pediatric cancer patients. Pediatr Hematol Oncol 1997; 14:547-54.

24 Solorzano-Santos F, Miranda-Novales MG, Diaz-Pena R, et al. Amikacin in single daily doses in children with fever. Revista de Investigacion Clinica 1996;48:13-18.

25 Nicolau DP, Freeman CD, Belliveau PP, Nightingale $\mathrm{CH}$, Ross JW, Quintiliani R. Experience with a once-daily aminoglycoside program administered to 2184 adult parts

26 Ho KK, Bryson SM, Thiessen JJ, Greenberg ML, Einarson TR, Leson CL. The effects of age and chemotherapy on gentamicin pharmacokinetics and dosing in pediatric oncology patients. Pharmacotherapy 1995;15:754-64.

27 Phillips JK, Spearing RL, Crome DJ, Davies JM. Gentamicin volumes of distribution in patients with hematologic disorders. N Engl F Med 1988;319:1290.

28 Zeitany RG, el Saghir NS, Santhosh-Kumar CR, Sigmon MA. Increased aminoglycoside dosage requirements in hematologic malignancy. Antimicrob Agents Chemother 1990;34:702-8.

29 Rodvold KA, Danzinger LH, Quinn JP. Single daily doses of aminoglycosides. Lancet 1997;350:1412.
30 Bertino JS Jr, Rotschafer JC. Single daily dose of aminoglycosides - a concept whose time has not yet come. 4:820-3

31 Karlowsky JA, Zhanel GG, Davidson RJ, Hoban DJ. Postantibiotic effect in Pseudomonas aeruginosa following single and multiple aminoglycoside exposures in vitro. $\mathscr{f}$ Antimicrob Chemother 1994;33:937-47.

32 Prins JM, Buller HR, Kuijper EJ, Tange RA, Speelman P. Once versus thrice daily gentamicin in patients with serious infections. Lancet 1993;341:335-9.

33 Chastagner P, Tourniaire B, Perel Y, et al. Prevention of gram-positive infections with teicoplanin during the induction therapy of childhood ALL: results of a prospective randomised trial. Med Pediatr Oncol 1997;29:334.

34 Lucas HP, Attard-Montalto S, Saha V, Bristow A, Kingston JE, Eden OB. Central venous catheter tip position and malfunction in a paediatric oncology unit. Pediatric Surgery International 1996;11:159-63.

35 Saha V, Gupta S, Daum RS. Occurrence and mechanisms of glycopeptide resistance in gram-positive cocci. Infect Agents glycopeptide resistance
Dis $1992 ; 1: 310-18$.

36 Centres for Disease Prevention and Control. Preventing the spread of vancomycin resistance: report from the hospital infection control practices advisory committee. Fed Reg

37 British Committee for Standards in Haematology. BCSH guidelines on the insertion and management of central venous lines. Br F Haematol 1997;98:1041-7.

38 Scholz M, Riesinger P, Reinhard H, Muller J, Graf N. Randomised comparison of two different time-based schedules in the treatment of infectious episodes in immunocompromised children. Med Pediatr Oncol 1997; 29:334.

39 Kumana CR, Yuen KY. Parenteral aminoglycoside therapy. Selection, administration and monitoring. Drugs 1994;47: 902-13. 